\title{
Santé précaire et transformation de la médecine du travail
}

Precarious health and changes in occupational medicine

\section{Gabrielle Lecomte-Ménahès}

\section{(2) OpenEdition}

\section{Journals}

Édition électronique

URL : http://journals.openedition.org/travailemploi/6490

DOI : 10.4000/travailemploi.6490

ISSN : 1775-416X

Éditeur

DARES - Ministère du Travail

\section{Édition imprimée}

Date de publication : 1 octobre 2014

Pagination : 35-47

ISSN : 0224-4365

\section{Référence électronique}

Gabrielle Lecomte-Ménahès, « Santé précaire et transformation de la médecine du travail », Travail et Emploi [En ligne], 140 | octobre-décembre 2014, mis en ligne le 01 octobre 2016, consulté le 20 avril 2019. URL : http://journals.openedition.org/travailemploi/6490 ; DOI : 10.4000/travailemploi.6490 


\title{
Santé précaire et transformation de la médecine du travail
}

\author{
Gabrielle Lecomte-Ménahès (*)
}

\begin{abstract}
À partir d'une enquête ethnographique et archivistique, cet article analyse un processus invisibilisé par la réforme en cours des services de santé au travail: la précarisation sanitaire des salariés rencontrés par les médecins du travail. La réorganisation et la démédicalisation des services provoquent la concentration des pratiques médicales sur ce public. Le suivi, plus long, des malades cantonne les médecins dans la gestion de problèmes de santé déclarés, ce qui les éloigne de leur mission première: empêcher la survenance d'accidents ou de maladies professionnels - mission désormais transférée aux professionnels pluridisciplinaires des services.
\end{abstract}

La loi de 2002 dite « de modernisation sociale ${ }^{(1)}$ » amorce une réforme orientant la médecine du travail vers la «santé au travail». Cette politique, qui passe par l'ouverture à la "pluridisciplinarité» des services de médecine du travail(2), se poursuit jusqu'aux décrets d'application ${ }^{(3)}$ parus en 2012. Les médecins du travail s'inquiètent de ces évolutions et s'interrogent sur l'avenir de leur profession et sur la répartition du travail de prévention entre les nouveaux professionnels recrutés et eux-mêmes. La focalisation sur la mise en place de la «pluridisciplinarité» et sur ses conséquences sur l'activité médicale occulte cependant un autre phénomène qui explique de manière tout aussi déterminante les changements de pratiques des médecins: la prise en charge croissante de salariés exposés à de mauvaises conditions d'emploi et de travail. La sociologie de la santé s'est largement intéressée à l'influence de la transformation du public sur les pratiques médicales: que ce soit par exemple à propos des maladies chroniques, que les médecins ne peuvent traiter comme des pathologies aiguës, ce qui met en difficulté le travail médical (BASZANGER, 1986) ou encore de «l'activisme thérapeutique» des malades du Sida (CARricaburu, Ménoret, 2005) avec lequel les médecins ont dû composer. Mais la médecine du travail, médecine de prévention auprès d'une population qui ne se définit pas par sa maladie et dont les praticiens ne peuvent prescrire de traitements, est un cas à part. Les rapports entre médecins et salariés s'apparentent davantage

(*) Centre nantais de sociologie; Gabrielle.LecomteMenahes@univ-nantes.fr

(1) Loi du 17 janvier 2002, n 2002-73, JO, 18 janvier 2002.

(2) Alors qu'historiquement ces services employaient des médecins du travail pour assurer la prévention de la santé des salariés, ils recrutent aujourd'hui des professionnels de diverses disciplines (hygiène, ergonomie, psychologie du travail, etc.). Voir la première partie de l'article pour plus de détails.

(3) Décret du 30 janvier 2012, n 2012-135, JO, 31 janvier 2012. à des relations de guichet - d'ailleurs les premiers sont fréquemment assimilés à des fonctionnaires par les seconds. Bien qu'exerçant majoritairement dans le secteur privé(4), ces médecins disposent du «pouvoir discrétionnaire» (SPIRE, 2008) propre aux agents des administrations. Ils décident, par le biais de l'avis d'aptitude, de qui peut travailler et de qui ne le peut pas. Comme l'a montré Michael LIPSKY (2010) dans Street-Level Bureaucracy à propos des fonctionnaires subalternes, au-delà d'une simple application de la règle, le contact avec le public amène les agents à redéfinir le contenu concret des politiques publiques qui ne sont alors plus seulement imposées «d'en haut» mais aussi négociées «par le bas». Les médecins du travail, comme les guichetiers de la caisse d'allocations familiales (DuBoIs, 2010) ou les médecins urgentistes des hôpitaux (PenefF, 1992), sont confrontés à une précarisation des conditions de vie ou de santé de leur public. La rencontre de salariés à la santé plus précaire ${ }^{(5)}$ et aux demandes spécifiques change alors les pratiques médicales. Bien que retenant l'attention des acteurs, l'introduction de la «pluridisciplinarité» n'est ainsi qu'un facteur parmi d'autres de la transformation en cours du métier de médecin du travail.

(4) «De manière relativement continue entre 1970 et 2010, environ neuf médecins du travail sur dix ont exercé dans le cadre d'un service interentreprises, couvrant une proportion similaire de salariés du secteur privé» (MARICHALAR, 2011, p. 105). Comme nous le verrons ensuite, les services interentreprises sont des associations d'entreprises employant des médecins du travail pour suivre la santé des salariés de leurs adhérentes. Les salariés de la fonction publique et du secteur agricole relèvent d'autres régimes de prévention de la santé au travail.

(5) Nous entendons ici par précaire ce «dont on ne peut garantir la durée, la solidité, la stabilité; qui, à chaque instant, peut être remis en cause» (Centre national de ressources textuelles et lexicales : http://www.cnrtl.fr/definition/pr\%C3\%A9caire; page consultée le 6 décembre 2014). 


\section{Encadré 1 \\ Méthodologie}

Pour comprendre la transformation de la médecine du travail, nous étudions les pratiques de ses acteurs historiques, les médecins, au sein des services «interentreprises» qui les emploient en majorité. Le suivi de la santé des salariés des entreprises adhérentes au service interentreprises est réparti entre les différents médecins du groupement. S'intéresser aux services interentreprises permet d'étudier les rencontres entre les médecins et un public salarié plus divers (par la taille des entreprises et leur secteur d'activité) que celui des services «autonomes ", qui emploient un ou plusieurs médecins au sein d'une seule et même entreprise de plus de 500 salariés.

L'approche ethnographique de cette recherche, réalisée dans le cadre d'une thèse en sociologie(1), permet de saisir «par le bas" les transformations de l'institution «médecine du travail » à travers les interactions de travail. Les données présentées sont issues de l'étude monographique d'un service de santé au travail interentreprises employant 150 personnes environ dont un peu moins d'une cinquantaine de médecins du travail. Ce service assure la surveillance de 122000 salariés travaillant dans les quelque 10000 entreprises adhérentes (2). L'enquête s'est déroulée de janvier 2011 à mars 2013. L'observation de réunions mensuelles de médecins du travail $(N=18)$ et la conduite d'entretiens semi-directifs avec divers acteurs du service ${ }^{(3)}$ nous ont aidée à comprendre le contexte de travail. Nous avons également observé des visites médicales $(N=157)^{(4)}$ en suivant plus particulièrement quatre médecins du travail. Le service étudié est implanté dans un département où l'offre de service prime sur les autres secteurs d'activité. Selon l'Insee, au 1 er janvier 2013, 64,4\% des établissements y relevaient du secteur “Commerce, transports et services divers" (et $21 \%$ étaient spécifiquement dédiés à l'automobile) (5). En 2011, ce secteur emploie environ $38 \%$ des salariés du département, devant celui «Administration publique, enseignement, santé, action sociale" qui en emploie $33 \%$ et l'industrie $18 \%\left({ }^{(6)}\right.$. Cette même année, les personnes en emploi sont majoritairement des employés et des ouvriers (ces deux catégories représentent chacune environ $27,5 \%$ du nombre total d'emplois), les professions intermédiaires occupent ensuite $24,3 \%$ des emplois et les cadres et professions intellectuelles supérieures $12 \%(7)$. Nous avons par ailleurs cerné l'évolution générale de l'activité médicale en effectuant des comptages à partir des rapports annuels d'activité du service. Ces rapports, produits par le médecin coordonnateur, comptabilisent les visites effectuées et saisies par les médecins ou leurs secrétaires dans un logiciel interne. Ils présentent la répartition des visites médicales selon la catégorisation du Code du travail de 2000 à 2012. Bien que cette catégorisation et les modes de saisie puissent varier d'un médecin à l'autre, les données ainsi collectées sont de meilleure qualité et davantage représentatives que celles produites par la Direction régionale des entreprises, de la concurrence, de la consommation, du travail et de l'emploi (Direccte) : les statistiques administratives régionales, dépendantes du nombre de rapports médicaux reçus et d'un mode de saisie très variable et hétérogène selon les années, comportent de trop nombreux biais pour être mobilisées.

Le recours aux méthodes archivistique et ethnographique permet d'avoir à la fois une vision globale de l'activité grâce au traitement des données chiffrées produites par les enquêtés et d'acquérir une connaissance des pratiques concrètes qu'enregistrent les catégories administratives ${ }^{(8)}$.

(1) Lecomte-Ménahès G., De la «médecine du travail» à la «santé au travail» : redéfinition d'une médecine de prévention, Thèse de doctorat en sociologie (en cours), sous la direction d'Annie Collovald et avec le coencadrement de Sarah Ghaffari, université de Nantes.

(2) Ces chiffres ont été arrondis.

(3) Cinq médecins du travail, trois intervenants en prévention des risques professionnels (IPRP), le directeur du service, quatre assistants de services de santé au travail (ASST) et deux secrétaires médicales nous ont accordé un entretien. Ceux que nous avons conduits avec les ASST et les secrétaires médicales sont des entretiens collectifs.

(4) Nous avons observé 49 visites périodiques, 56 visites d'embauche, 27 visites de reprise, 5 visites de préreprise et 20 visites occasionnelles.

(5) Insee, Répertoire des entreprises et des établissements (Sirène).

(6) Insee, Recensement de la population 2011, exploitations complémentaires lieu de travail.

(7) En 2011, 65,7\% de la population de 15 à 64 ans de ce département est active occupée, $8,1 \%$ est au chômage et 26,2\% est inactive, selon l'Insee (Recensement de la population, exploitations principales).

(8) Sur ce point, voir Lomba (2008).

L'article s'organise en trois points. Après avoir décrit les enjeux de l'introduction de la «pluridisciplinarité» dans les services de santé au travail interentreprises dans une première partie, nous nous concentrerons dans un deuxième temps sur la réorganisation de l'activité médicale, marquée à la fois par un suivi plus irrégulier de l'ensemble de la population salariée et une attention plus grande accordée à celle exposée à de mauvaises conditions d'emploi et de travail. Nous verrons enfin que cette réorganisation induit un ajustement des pratiques de travail des médecins au public rencontré lors de visites médicales spécifiques (voir encadré 1 pour la méthodologie de notre enquête).

\section{L'ouverture à la "pluridisciplinarité"}

\section{De la médecine du travail à la santé au travail}

Depuis la fin du XIXe siècle, la gestion assurantielle française de la santé au travail rend non seulement 
les employeurs responsables des risques auxquels ils exposent leurs salariés mais les contraint à les en protéger (BRUNo et al., 2011). Cette protection s'est institutionnalisée, suite à la seconde guerre mondiale, avec le suivi médical des salariés (Buzzi et al., 2006) : les entreprises de plus de 500 salariés doivent désormais employer leur propre médecin du travail au sein d'un service autonome tandis que les autres peuvent adhérer à un service interentreprises.

Depuis le début des années 1970, la politique patronale de gestion de la santé au travail s'oriente vers la démédicalisation et la valorisation de la «pluridisciplinarité» (MARICHALAR, 2010). Elle se concrétise par la promulgation de la loi du 17 janvier $2002^{(6)}$ qui débaptise les services de «médecine du travail» pour les renommer services de «santé au travail». Ce nouveau nom symbolise l'introduction de la «pluridisciplinarité», notamment dans les services interentreprises où jusqu'alors les médecins disposaient d'un quasimonopole de l'activité de prévention. Seuls quelques hygiénistes y exerçaient également depuis une vingtaine d'années. Le décret du 24 juin $2003^{(7)}$ a permis de recruter des ergonomes, des hygiénistes ou des psychologues du travail, détenteurs de l'habilitation d'«intervenants en prévention des risques professionnels» (IPRP). Puis la loi du 20 juillet $2011^{\left({ }^{(8)}\right.}$ a acté l'emploi d'infirmiers et «d'assistants de services de santé au travail» (ASST).

Ce changement de dénomination s'accompagne d'un transfert des « fondements constitutifs » de l'institution, selon le sens que donne Anselm Strauss à l'expression (1992, p. 95). Pour StRauss, ces fondements correspondent à un «objectif vaguement ambigu» sur le rôle de l'institution qui, accepté par l'ensemble de ses membres, n'est jamais remis en question. Ils ont trait ici à la mission de prévention des services interentreprises. Historiquement attribuée aux seuls médecins du travail, cette mission est instituée par la loi de 1946 dans les termes suivants : «ces services seront assurés par un ou plusieurs médecins qui prennent le nom de "médecins du travail" dont le rôle exclusivement préventif consiste à éviter toute altération de la santé du travailleur du fait de son travail ${ }^{(9)}{ }_{\gg}$. La loi du 20 juillet 2011 étend l'application de ces principes aux services de santé au travail dans leur ensemble ${ }^{(10)}$, actant ainsi l'élargissement des pratiques de prévention à divers groupes professionnels.

(6) Loi du 17 janvier 2002, $\mathrm{n}^{\circ}$ 2002-73, op. cit.

(7) Décret du 24 juin 2003, n 2003-546, JO, 26 juin 2003.

(8) Loi du 20 juillet 2011, $\mathrm{n}^{\circ}$ 2011-867, article L. 4622-8, JO, 24 juillet 2011.

(9) Loi du 11 octobre 1946, $\mathrm{n}^{\circ}$ 46-2195, article 1, JO, 12 octobre 1946.

(10) «Les services de santé au travail ont pour mission exclusive d'éviter toute altération de la santé des travailleurs du fait de leur travail.» (Loi du 20 juillet 2011, n 2011-867, article L. 4622-2, op. cit.) On notera que le «travailleur» est mis au pluriel, marquant la volonté d'une approche collective de la prévention.
Ces dernières sont généralement divisées en plusieurs segments. La prévention primaire est destinée à empêcher la survenance d'accident ou de maladie; la prévention secondaire vise à dépister les maladies professionnelles avant que n'apparaissent des signes cliniques et la prévention tertiaire consiste à soigner des problèmes de santé déjà bien identifiés. La prévention primaire qui, comme nous allons le voir, est transférée aux acteurs pluridisciplinaires, est plus valorisée que la prévention tertiaire pratiquée majoritairement par les médecins. Une telle conception est notamment véhiculée par le Centre interservices de santé et de médecine du travail en entreprise (Cisme), qui représente les services interentreprises de santé au travail :

"Alors que j'attends un rendez-vous avec le directeur d'un service de santé au travail interentreprises, je remarque dans le hall d'entrée une affiche du Cisme accrochée près de la porte. Il s'agit d'un schéma récapitulant l'évolution de la "médecine du travail" à la "santé au travail" et les bénéfices apportés par ce changement. Un point particulier attire mon attention, il y est écrit qu'hier il s'agissait d'un système de prévention tertiaire, qu'aujourd'hui il s'agit de prévention secondaire, et que demain il s'agira de prévention primaire. L'affiche se trouve également dans le bureau du directeur, près de la porte d'entrée. »

(Notes de terrain, observation réalisée le 13 octobre 2010.)

Cette vision évolutionniste de la santé au travail montre le "progrès» de la prévention suivant ce modèle.

\section{Une division du travail de prévention}

Les préventions de type primaire, secondaire et tertiaire se sont toujours entremêlées dans les pratiques des médecins du travail. L'entretien et l'examen clinique leur permettent d'apprécier l'état sanitaire du salarié et de l'articuler à la connaissance de ses conditions de travail. Lors des visites médicales, les salariés dont la santé est plus ou moins atteinte exposent aux médecins des problèmes auxquels ceux-ci tentent ensuite d'apporter une solution en se rendant dans les entreprises afin d'y faire modifier les conditions collectives de travail. Mais l'actuelle surcharge de travail médical, due, comme nous le verrons, à la «pénurie» de médecins du travail et à l'accueil de plus en plus fréquent d'un public à la santé précaire, contraint les médecins à se concentrer sur cette catégorie de travailleurs auprès de laquelle ils font de la prévention tertiaire, de sorte qu'ils ont moins de temps à consacrer à la prévention primaire qui est alors déléguée aux professionnels «pluridisciplinaires». Une nouvelle division du travail, qui segmente les groupes professionnels selon les niveaux de prévention, se met alors en place dans les services de santé au travail interentreprises. 


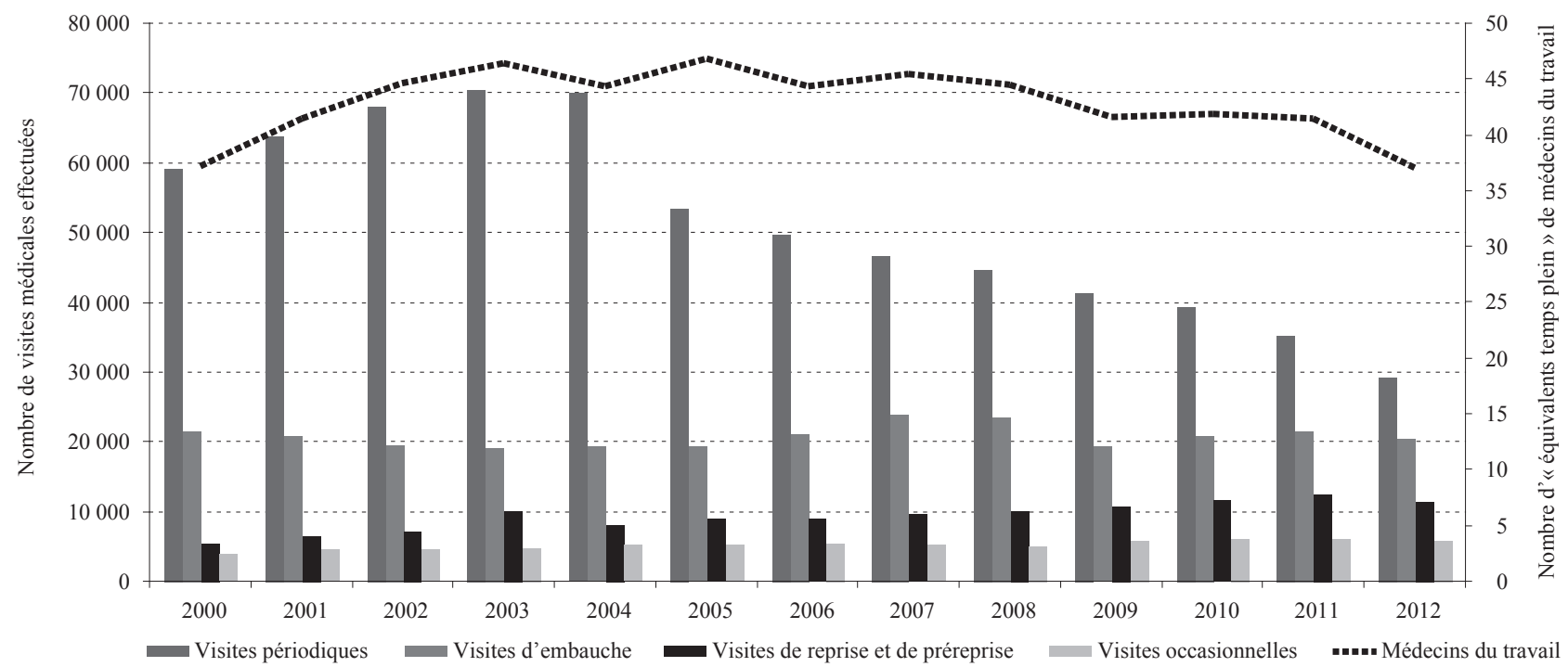

Lecture : En 2010, 39533 visites périodiques ont été effectuées au sein du service qui employait alors 41,8 « équivalents temps plein » de médecins du travail.

Champ : Visites médicales effectuées au sein du service étudié.

Source : Rapports annuels du service étudié (2000-2012).

Les «entretiens infirmiers» viennent se substituer aux visites périodiques des médecins pour assurer un suivi sanitaire régulier des salariés. Les infirmiers y recueillent des informations sur leur santé et leur travail et leur signalent les risques auxquels ils sont exposés. Ils repèrent les éventuels problèmes et redirigent les travailleurs concernés vers les médecins auxquels ils sont associés. Ce suivi, grâce aux informations données par les salariés, permet d'apprécier les contextes de travail.

Selon leurs spécialités, les intervenants en prévention des risques professionnels évaluent différents risques dans les entreprises adhérentes et les conseillent sur la manière de faire de la prévention. Les employeurs, en tant que producteurs de risques, ont en effet un rôle fondamental à jouer dans la prévention primaire. Les assistants de services de santé au travail prennent, quant à eux, en charge un premier repérage des risques au sein des plus petites entreprises, qui aboutit souvent à la rédaction d'une première version de la fiche d'entreprise ${ }^{(11)}$. On considère que l'évaluation des risques et la prévention des infirmiers, intervenants et assistants relèvent d'une prévention de type primaire car elles cherchent à limiter l'exposition des salariés.

Le travail de prévention qu'hier les médecins faisaient pratiquement seuls est donc aujourd'hui réparti entre plusieurs catégories de professionnels. Cette division du travail et la délégation de la prévention primaire qui en découle sont facilitées par le fait que les médecins sont accaparés par le suivi d'un public à la santé précaire.

(11) La fiche d'entreprise, transmise à l'employeur, récapitule les risques professionnels repérés et les effectifs de salariés qui y sont exposés.

\section{La réorganisation de l'activité des médecins du travail : vers une prise en charge accrue de la santé précaire...}

\section{Un suivi plus irrégulier des salariés}

L'activité des médecins du travail s'articule autour des visites médicales. Selon le sociologue Daniel BACHet (2011, p. 55), les médecins étaient historiquement "focalisés sur la visite annuelle systématique», c'est-à-dire sur un type de visite qualifiée de «périodique» par les acteurs euxmêmes. À partir de 1952, ils pratiquent ainsi une médecine de «visites à la chaîne» permettant la remise d'avis d'aptitude (Buzzi et al., 2006, p. 66). Bien qu'ils débattent de l'utilité des visites médicales systématiques, celles-ci constituent toujours leur principale activité. Cependant, les comptages réalisés à partir des rapports d'activité du service étudié mettent en lumière une diminution de moitié du nombre des visites médicales périodiques de 2000 à 2012 (voir graphique 1) par opposition à des visites «non périodiques» qui comprennent les visites d'embauche, de reprise ${ }^{(12)}$ et de préreprise ${ }^{(13)}$ ainsi que les autres visites dites

(12) «Le salarié bénéficie d'un examen de reprise du travail par le médecin du travail : après un congé de maternité; après une absence pour cause de maladie professionnelle; après une absence d'au moins trente jours pour cause d'accident du travail, de maladie ou d'accident non professionnel.» (Décret du 30 janvier 2012, n 2012-135, article R. 4624-22, op. cit.) (13) «En vue de favoriser le maintien dans l'emploi des salariés en arrêt de travail d'une durée de plus de trois mois», les visites de préreprise ont lieu pendant l'arrêt de travail. (Décret du 30 janvier 2012, $\mathrm{n}^{\circ}$ 2012-135, articles R. 4624-20 et R. 4624$21, J O, 31$ janvier 2012.) 
«occasionnelles». Cette transformation remet en cause le suivi systématique de l'ensemble des salariés.

Au-delà des seules visites périodiques, l'ensemble des visites médicales effectuées au sein du service décroît de $25 \%$ de 2000 à 2012 . Cette baisse s'explique par les diminutions du nombre de médecins et du temps de travail médical au sein du service. Après avoir employé 37,2 «équivalents temps plein» $(\mathrm{ETP})^{(14)}$ de médecins en 2000 puis 46,9 en 2005, environ 37 ETP de médecins sont salariés en 2012 (voir graphique 1). Ces effectifs correspondent à ce que l'on peut trouver au sein de la population générale des médecins du travail, qui est, selon les acteurs du champ de la santé au travail, affectée par un «déficit médical». Elle est en effet vieillissante et recrute peu(15). Mais cette diminution n'entraine pas pour autant celle du nombre de salariés suivis. Au sein du service présenté ici, un médecin à temps plein devait théoriquement suivre 2580 salariés en 2005 et 580 de plus en $2012^{(16)}$.

L'année 2004 marque une rupture dans l'évolution des visites périodiques pratiquées au sein $\mathrm{du}$ service enquêté. Après une augmentation de $18,1 \%$ de 2000 à 2004 , elles baissent brutalement de $23,3 \%$ entre 2004 et 2005 et fléchissent continûment depuis. Le décret du 28 juillet $2004(17)$ modifie les articles du Code du travail encadrant la périodicité de ces visites qui, d'annuelle, devient biennale. Par ce texte, les législateurs cherchent à réduire la charge de travail des médecins qui peinent à suivre l'ensemble des effectifs salariés qui leur sont attribués. Le «déficit médical» est, par ailleurs, un argument récurrent des directions de services interentreprises pour réformer la médecine du travail (MARICHALAR, 2010) et pour accroître le nombre de salariés suivis par chaque médecin.

Cette révision des textes de loi n'est pas la seule raison du suivi plus irrégulier des salariés. Bien que les délais d'obtention des visites médicales soient plus longs, l'accroissement des visites non périodiques qui sont souvent effectuées en priorité et qui demandent davantage de travail aux médecins

(14) De nombreux médecins du travail exerçant à temps partiel, leur temps et leur charge de travail sont souvent comptés en "équivalent temps plein», ce qui permet leur comparaison.

(15) Il est cependant difficile de connaître précisément la démographie de cette population. Sur cette question, voir la thèse de Pascal MarichaLAR (2011) et plus particulièrement les pages 80 à 93 .

(16) Le département du service étudié connaît un taux d'emploi relativement stable qui passe de $66 \%$ en 2006 à $65,7 \%$ en 2011 (Insee, Recensement de la population, exploitations principales). L'augmentation du nombre de salariés «attribués» à chaque médecin du travail est avant tout à relier au «déficit médical».

(17) Décret du 28 juillet 2004, $\mathrm{n}^{\circ}$ 2004-760, JO, 30 juillet 2004. explique également leur impossibilité à rencontrer l'ensemble des travailleurs. Ainsi, les visites «non périodiques» augmentent de $22,4 \%$ de 2000 à 2012 au sein du service, malgré le fléchissement du nombre total de visites. Après s'être accrues de $30,4 \%$ de 2000 à 2011 , elles chutent de 8 points entre 2011 et 2012, suivant la baisse brutale du nombre de médecins du travail employés par le service (voir graphique 1). Sur l'ensemble des visites médicales, la part des «non-périodiques» est passée de 34,4\% en 2000 à 56,4\% en 2012 au sein du service ${ }^{(18)}$. Cette nouvelle répartition de l'activité médicale amène les médecins du travail à prendre davantage en charge des publics à la santé précaire.

\section{Des différences de public selon les visites}

Au-delà des différents intitulés administratifs qui ne nous renseignent pas sur la réalité des situations vécues par les salariés, l'augmentation des visites d'embauche, de préreprise ou de reprise après arrêt de travail indique tout de même que les médecins se trouvent plus souvent face à des travailleurs à la santé précaire du fait de leur exposition à de mauvaises conditions d'emploi et de travail. Les chercheurs ont souvent recherché les causes de la précarité dans les formes d'emploi (CASTEL, 1999) mais c'est oublier le rôle fondamental du rapport entre la santé et les conditions de travail, pardelà les statuts d'emploi (ThÉBAud-Mony, 1997; CélérIer, 2008). Pour Valentine Hélardot (2005, p. 31), ce sont en effet les «mutations conjuguées de l'emploi et du travail» qui renforcent les effets pathogènes du travail et complexifient sa conciliation avec la santé pour une part croissante de salariés. L'auteure montre que la précarisation des salariés est liée au développement des contrats atypiques, mais aussi à la reconfiguration des relations salariales s'éloignant du modèle salarié/ employeur, à l'intensification du travail ainsi qu'à la sollicitation des subjectivités individuelles par le néo-management. Les liens entre santé précaire et précarité professionnelle sont de fait complexes, ce dont rendent compte les différents types de visites que nous avons observées.

Hélardot (2008, p. 78) note que «l'intensification du travail ravive des enjeux classiquement connus quant à la santé et à la sécurité des salariés sur les lieux de travail. [...] Outre les sollicitations corporelles et mentales directes, [elle] atteint également la santé en réduisant les marges de manœuvre laissées aux salariés dans la réalisation concrète $\mathrm{du}$ travail». Les salariés qui cumulent emplois précaires et mauvaises conditions de travail

(18) La part des visites d'embauche augmente de 6,5 points, celle des visites de reprise et de préreprise de 11,1 points et la part des visites occasionnelles de 4,5 points de 2000 à 2012 . 
(Gollac, Volkoff, 2007) sont les plus exposés ${ }^{(19) .}$ Ceux encourant des risques particuliers bénéficient d'une «surveillance médicale renforcée», mais ces visites périodiques supplémentaires ne sont souvent plus effectuées par les médecins, surchargés de travail. C'est plutôt lors des visites de reprise après un arrêt de travail que les salariés les rencontrent. Au sein du service étudié, ce public est majoritairement composé d'employés et d'ouvriers ${ }^{(20)}$ dont la moitié a plus de 48 ans. Or «les ouvriers sont plus souvent absents pour des raisons de santé $(4,5 \%$ en moyenne sur la période étudiée) que les cadres (1,6\%). [...] Ces différences demeurent significatives "toutes choses égales par ailleurs" [et] tendent à se creuser avec l'âge : entre 55 et 59 ans l'écart d'absentéisme entre cadres et ouvriers atteint 5 points, alors même qu'une fraction importante des ouvriers dont la santé est la plus dégradée a déjà quitté le marché du travail» (InAN, 2013, p. 4). En outre, «l'absentéisme augmente fortement avec le niveau d'exposition aux contraintes physiques et psychosociales» (ibid, p. 5).

$\mathrm{Au}$ sein du service présenté ici, les visites de reprise augmentent de $82 \%$ de 2000 à $2012^{(21)}$. Leur croissance renvoie surtout à celle des visites après arrêt de maladie qui représentent environ $60 \%$ de l'ensemble. Les congés de maternité mis à part, cet accroissement a pour conséquence de davantage confronter les médecins du travail à une population à la santé dégradée. La durée légale de l'arrêt à l'issue duquel une visite de reprise doit avoir lieu a été allongée à trente jours ${ }^{(22)}$, ce qui place les médecins du travail face aux salariés aux arrêts les plus longs. Les médecins se plaignent d'ailleurs de perdre contact avec les autres salariés arrêtés, et craignent de méconnaître de nombreuses situations délétères.

$\mathrm{Au}$ contraire de salariés aux contrats de travail plus précaires que leurs problèmes de santé feraient sortir de l'emploi, ceux rencontrés lors

(19) Les arrêts de travail suite à des maladies professionnelles et des accidents du travail sont des conséquences marquantes des risques professionnels sur la santé. Cependant, les médecins enquêtés insistent également sur l'importance de problèmes de santé, moins visibles, en lien avec le travail, que les salariés omettent de signaler et qu'eux diagnostiquent, comme par exemple des déficiences auditives dues à un environnement de travail bruyant.

(20) Ils sont : carrossier, assistante maternelle, couvreur, agent de sécurité, agent d'entretien des espaces verts, commerciale, agent d'entretien, pâtissier, chauffeur, aide soignante, veilleuse de nuit, mécanicien, magasinier, cuisinière, opérateur, assistante juridique, technicien, aide à domicile.

(21) Après que les visites de reprise ont augmenté de 109,4\% entre 2000 et 2011, nous notons une diminution de 27,4 points entre 2011 et 2012. Nous la rapportons à la baisse du nombre total de visites sur cette période, liée au «déficit» médical.

(22) Elle était de 8 jours après accident du travail et 21 jours après maladie ou accident non professionnel avant d'être modifiée par le décret du 30 janvier 2012, n 2012-135, article R. 4624-22, op. cit. des visites de reprise ont un emploi stable qu'ils retrouvent à l'issue de leur arrêt de travail. Mais «peuvent [...] se retrouver aussi précarisés des travailleurs du noyau stable des entreprises, du fait des transformations de l'organisation du travail qui les contraint, ou du fait des modifications du rapport à leur travail liées aux mécanismes de sous-traitance» (HuEz, 1997, p. 18). Ces salariés doivent concilier santé et travail, ce qui devient parfois plus difficile au cours de la carrière professionnelle. HÉlaRdot (2008, p. 79) fait d'ailleurs valoir que «dans le contexte d'un marché de l'emploi hyperconcurrentiel, et face à des exigences toujours plus fortes dans l'accomplissement du travail, les salariés dont la santé est défaillante sont les premiers à voir leur intégration professionnelle fragilisée». Les salariés qui ont les arrêts de travail les plus longs et souvent les problèmes de santé les plus graves rencontrent les médecins du travail en visites de préreprise lors desquelles l'inaptitude est régulièrement envisagée.

Plusieurs mesures de politiques publiques ont été prises pour maintenir dans l'emploi la population salariée vieillissante. La visite de préreprise est rendue obligatoire par le décret $n^{\circ}$ 2012-135 du 30 janvier 2012. Le nombre de ces visites pour 100 salariés a quintuplé au sein du service étudié entre 2000 et 2012, passant de $0,4 \%$ à $2 \%$. Suivant la politique de prévention de la désinsertion professionnelle de la Caisse nationale de l'assurance-maladie des travailleurs salariés (Cnam-TS) instaurée en $2009{ }^{(23)}$, les médecins conseils de la Sécurité sociale(24) sollicitent davantage ce type de visite depuis cette date. Sur l'ensemble des visites de préreprise, la part de celles à l'initiative des médecins conseils a augmenté de 4,1 points entre 2000 et 2009 et de 13,9 points de 2009 à 2012, devançant celle des visites sollicitées par les médecins traitants qui a diminué de 4,2 points. Les visites à la demande des salariés restent cependant largement majoritaires (voir graphique 2).
(23) Une des recommandations de la Convention d'objectifs et de gestion pour la branche AT/MP 2009-2012 publiée par la Cnam-TS est de «développer les relations entre médecins traitants, médecins conseils, et médecins du travail, notamment pour le signalement précoce des cas de risque de désinsertion afin d'augmenter le nombre de visites de préreprise». Voir : CNAM-TS (2009), «Programme 6, Prévention de la désinsertion professionnelle», Convention d'objectifs et de gestion pour la branche AT/MP 2009-2012, Paris, p. 32; consulté le 14 mars 2014 sur http://www.risquesprofessionnels.ameli.fr/filead$\mathrm{min} /$ user upload/document PDF a telecharger/COG\%20 $2009-2012 \% 20$ Assurance $\% 20$ Maladie $\% 20-\% 20$ Risques $\% 20$ Professionnels.pdf

(24) Les médecins conseils, employés par les caisses d'assurance-maladie, donnent leur avis médical pour la prise en charge par la Sécurité sociale de prestations associées à la reconnaissance d'accidents du travail et d'invalidités. Ils statuent sur les situations de salariés suivis par les médecins du travail. 
Graphique 2 : Nombre de visites de préreprise de 2000 à 2012

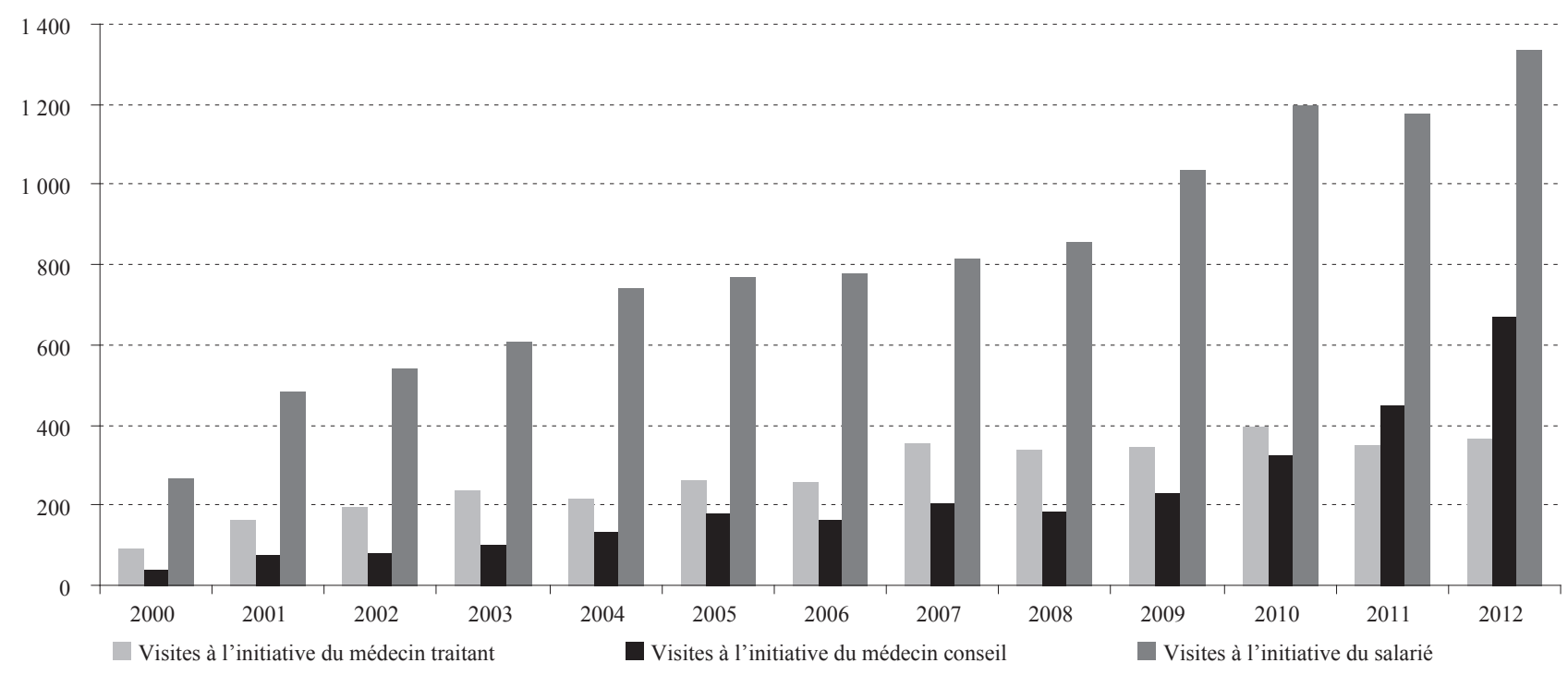

Lecture : 670 visites de préreprise demandées par un médecin-conseil de la Sécurité sociale ont été effectuées au sein du service en 2012. Champ : Visites médicales de préreprise effectuées au sein du service étudié.

Source : Rapports annuels du service étudié, (2000-2012).

Ces demandes des salariés sont à mettre en lien avec la médiatisation croissante du stress au travail depuis les années 1990 (LorioL, 2011). Les « risques psychosociaux ${ }^{(25)}$ » sont devenus des enjeux politiques et de recherche qui favorisent l'expression des problèmes personnels des salariés. En 2010, sur l'ensemble des visites occasionnelles du service que nous avons étudié, la part (majoritaire) des visites «à la demande du salarié » est de 44,6\%. Ces visites augmentent de $33,6 \%$ entre 2000 et 2010 . Les salariés les sollicitent largement pour des problèmes de «souffrance au travail( ${ }^{(26)}$ ».

Selon Valentine Hélardot (2008), la précarisation de l'emploi, induisant une irrégularité de revenus, rend la préservation quotidienne de la santé plus difficile. Les médecins du travail rencontrent ainsi en visite d'embauche des salariés, souvent âgés d'une vingtaine d'années, aux formes d'emploi les plus instables ${ }^{(27)}$. Notons «[qu'en 2009] la moitié des salariés embauchés en CDD, stage ou

(25) Le Collège d'expertise sur le suivi des risques psychosociaux au travail les définit comme «risques pour la santé mentale, physique et sociale, engendrés par les conditions d'emploi et les facteurs organisationnels et relationnels susceptibles d'interagir avec le fonctionnement mental» (GolLaC, 2012, p. 7).

(26) C'est ainsi que les définissent de nombreux médecins du travail reprenant le concept du psychiatre et psychanalyste Christophe Dejours (1998).

(27) «Les CDD et missions d'intérim, souvent de très courte durée, participent au fort taux de rotation de l'emploi et sont même très majoritaires dans les flux d'embauche. Ainsi, selon les déclarations uniques d'embauche (hors mission d'intérim) de l'Acoss, près de $80 \%$ des intentions d'embauche de l'année 2006 correspondent à des CDD et, parmi ceux-ci, les deux tiers sont des CDD de moins d'un mois (soit 9 millions). La même année, plus de 16 millions de contrats d'intérim ont été conclus : $88 \%$ d'entre eux pour moins d'un mois et un quart pour une seule journée» (LAGARENNE, LAMARCHE, 2008, p. 51). apprentissage ont moins de 29 ans [...]. D'ailleurs, parmi les moins de 25 ans, la part de ces formes particulières d'emploi est de 49,7\%» (MANUY, NouËL DE LA BuzonNière, 2011, p. 1). La part des visites d'embauche sur l'ensemble de celles effectuées au sein du service étudié diminue de 5,6 points entre 2000 et 2003, puis augmente de 12,1 points de 2003 à $2012^{(28)}$.

\section{-..Qui induit une transformation de leurs pratiques de travail}

\section{Renforcement du rôle de suivi des malades}

La précarisation de la santé du public rencontré conduit les médecins du travail à prendre en charge davantage de malades. Au cours de nos observations, nous avons constaté que les médecins consacraient une part importante de leur «tiers-temps ${ }^{(29)}$ », dédié à l'action en entreprise, à tenter de résoudre les situations problématiques de certains salariés. Ces «cas», qui les occupent durant plusieurs semaines, les confrontent à une temporalité différente des

(28) Les «emplois temporaires (CDD, missions d'intérim...) prennent de plus en plus d'importance dans le secteur privé ou semi-public. [...] Entre 1990 et 2007, selon les recensements de la population, la part des emplois temporaires dans l'emploi salarié est passée de $10 \%$ à $15 \%$ » (MARCHAND, 2010, p. 4). Cette augmentation des formes atypiques d'emploi est à corréler à leur meilleure prise en compte au sein des entreprises et des services de santé au travail, au sein desquels un médecin est parfois dédié au suivi des intérimaires.

(29) «L'employeur ou le président du service interentreprises prend toutes mesures pour permettre au médecin du travail de consacrer à ses missions en milieu de travail le tiers de son temps de travail, dans le cadre des actions mentionnées à l'article R. 4624-1.» (Décret du 30 janvier 2012, n 2012-135, article R. 4624-4, op. cit.) 
vacations où les visites s'enchaînent les unes aux autres. Est par ailleurs exigé d'eux un rôle plus thérapeutique, puisqu'il s'agit de tenter de soulager la souffrance qui leur est exposée ${ }^{(30)}$ : la visite de préreprise, et tout le travail médical de préparation de l'inaptitude qui en découle généralement, en attestent.

Faisant l'histoire de l'inaptitude au travail, Catherine OMNÈs montre qu'après avoir concerné des travailleurs usés par une longue et difficile carrière, de l'après-guerre au début des années 1970, elle touche désormais des travailleurs aux parcours professionnels beaucoup plus fragmentés, constituant ainsi le «statut des exclus»: en effet, «[l'inaptitude] a drainé vers elle les fractions les plus fragilisées de la société dont les parcours médicaux, professionnels et familiaux témoignent beaucoup plus des effets cumulatifs de l'usure des conditions de travail et de vie que des accidents biographiques » (OMNÈs, BRUNO [dir.], 2004, p. 203).

L'inaptitude au travail interdit l'emploi du salarié à un poste de travail afin de protéger sa santé. Elle débouche régulièrement sur un licenciement. Aujourd'hui, l'inaptitude accompagne la gestion des fins de carrière ${ }^{(31)}$ d'une partie des salariés dont la santé a été dégradée par l'exposition à de mauvaises conditions de travail et d'emploi. Quand elle a lieu, et au terme de difficiles périodes de transition, la réinsertion professionnelle des personnes inaptes nécessite souvent qu'ils obtiennent le statut de travailleurs handicapés afin d'accéder à des dispositifs de reconversion spécifiques.

En 2004, «environ 6\% des salariés examinés annuellement par la médecine du travail ont des problèmes d'aptitude soit probablement 700000 personnes chaque année» (ibid, p. 10). Mais cette estimation ne rend pas compte de l'accroissement récent des déclarations d'inaptitude délivrées par les médecins du travail. Au sein du service étudié, sur l'ensemble des avis rendus, la part des aptitudes diminue de 5 points de 1985 à 2011 alors que les inaptitudes définitives augmentent de 0,8 point sur la même période ${ }^{(32)}$ (voir tableau). Notons que ces déclarations ne recouvrent pas l'ensemble des salariés à la santé précaire; en effet, l'inaptitude ne peut pas être pour tous un moyen de reconversion professionnelle et nombreux sont ceux qui préfèrent, quand ils le peuvent en évitant d'être déclarés

(30) Particulièrement dans les cas de «souffrance au travail» où l'écoute fait partie du travail thérapeutique.

(31) Au sein du service étudié, les trois quarts des salariés observés déclarés inaptes ont plus de 39 ans et la moitié plus de 52 ans. Les deux salariés de moins de quarante ans l'ont été pour des problèmes de «souffrance au travail».

(32) Dans ce service, une vingtaine d'avis d'inaptitude définitive ont été rendus en 1985 et environ 630 en 2011, soit près de trente fois plus. inaptes, conserver un emploi délétère plutôt que devenir inactifs ${ }^{(33)}$.

Tableau : Répartition des avis d'aptitude de 1985 à 2011 au sein du service étudié (en pourcentage)

\begin{tabular}{|l|c|c|c|c|c|c|}
\hline & $\mathbf{1 9 8 5}$ & $\mathbf{1 9 9 0}$ & $\mathbf{1 9 9 5}$ & $\mathbf{2 0 0 0}$ & $\mathbf{2 0 0 5}$ & $\mathbf{2 0 1 1}$ \\
\hline Avis d'aptitude & 95,9 & 94,4 & 94,1 & 96,2 & 93,7 & 90,9 \\
\hline Avis d'aptitude limitée & 3,6 & 4,7 & 4,8 & 2,8 & 4,6 & 6,8 \\
\hline Avis d'inaptitude temporaire & 0,4 & 0,7 & 0,7 & 0,5 & 1,1 & 1,3 \\
\hline Avis d'inaptitude définitive & 0,1 & 0,2 & 0,3 & 0,5 & 0,6 & 0,9 \\
\hline $\begin{array}{l}\text { Ensemble des avis d'aptitude/ } \\
\text { inaptitude }\end{array}$ & 100 & 100 & 100 & 100 & 100 & 100 \\
\hline
\end{tabular}

Lecture : En 2005, les avis d'aptitude limitée (avis d'aptitude avec restrictions, avec demandes d'aménagement du poste de travail, « à revoir » ou inaptitude à un poste mais aptitude à un autre) représentaient 4,6\% de l'ensemble des avis d'aptitude et d'inaptitude délivrés au sein du service étudié

Champ : Avis d'aptitude et d'inaptitude délivrés dans le service étudié de 1985 à 2011.

Source : Rapports annuels du service étudié $(1985 ; 1990 ; 1995$; $2000 ; 2005 ; 2011)^{(34)}$.

Tout comme les agents d'accueil des caisses d'allocations familiales étudiés par Vincent DuBoIs, les médecins du travail sont confrontés à la précarisation du public qu'ils prennent en charge, ici plus particulièrement à celle de leur santé. L'arrivée d'une population aux situations «hors normes», "génératrice d'incertitude et d'inquiétude, est particulièrement propice à l'activation de dispositions personnelles des guichetiers dans leur travail ou, pour le dire comme Michel Dobry, "à la régression vers les habitus" ${ }^{(35)} \gg$ (DuBoIs, 2010, p. 108). Chez les médecins du travail, que leur formation initiale prépare davantage à la guérison qu'à la prévention, elle se traduit par une concentration de leur activité sur la prise en charge des malades auprès desquels ils pratiquent une prévention «tertiaire».

(33) À état de santé égal, une négociation plus ou moins explicite peut s'établir entre le médecin et le salarié de sorte à favoriser ou éviter l'inaptitude. Connaissant les conséquences sur l'emploi de ce type de décision, les médecins du travail sont nombreux à prendre en compte l'avis du salarié avant de le déclarer inapte. Voir l'exemple présenté dans l'encadré 3 où la salariée préfère conserver un emploi pour lequel la médecin du travail pourrait la déclarer inapte afin de ne pas se retrouver sans activité professionnelle.

(34) N'ayant pu obtenir les données de l'année 2010, nous avons choisi de prendre l'année 2011 comme référence.

(35) Il fait ici référence au titre du septième chapitre de l'ouvrage de Michel Dobry (1986) où celui-ci discute l'idée selon laquelle les conjonctures de crise sont propices à l'ouverture de choix plus étendus pour les individus; il émet l'hypothèse qu'au contraire, dans ces moments-là, leurs perceptions et leurs comportements restent façonnés par leurs habitus. Le terme «habitus» se rapporte au concept développé par Pierre BourdiEu (2000, p. 256) qui l'entend comme un «système de dispositions durables, structures structurées prédisposées à fonctionner comme des structures structurantes, c'est-à-dire en tant que principe de génération et de structuration de pratiques et de représentations qui peuvent être objectivement "réglées" et "régulières" sans être en rien le produit de l'obéissance à des règles ». 
Graphique 3 : Répartition des avis d'aptitude avec restrictions ou aménagement et des avis d'inaptitude selon la catégorie de visite médicale en 2006

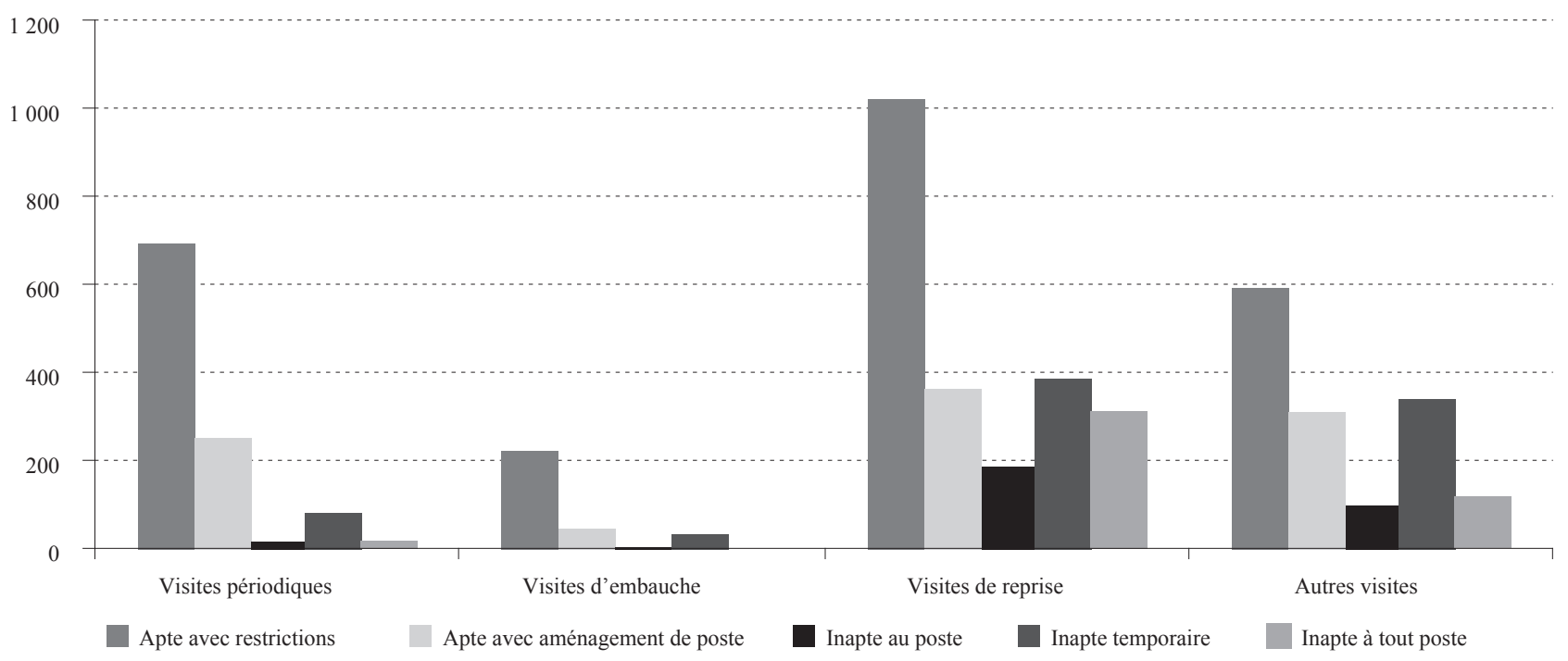

Lecture : En 2006, 309 avis d'inaptitude à tout poste de travail dans l'entreprise ont été délivrés en visites de reprise dans le service étudié. Les visites de préreprise sont comptabilisées dans « autres visites ».

Champ : Avis d'aptitude avec restriction ou aménagement de poste et avis d'inaptitude délivrés dans le service étudié en 2006.

Source : Rapport annuel du service étudié (2006).

\section{Des pratiques ajustées aux catégories administratives}

Les déclarations d'inaptitude définitive suivent un protocole particulier qui nécessite un travail administratif et de «tiers-temps». Selon le Code du travail(36), le médecin ne peut délivrer ce type d'avis qu'après avoir réalisé une étude du poste et des conditions de travail du salarié entre deux examens médicaux espacés de deux semaines. Des échanges avec l'employeur, qui doit lui aussi faire des propositions d'aménagement du poste de travail, sont nécessaires. À la déclaration d'inaptitude, s'ajoute souvent un travail administratif d'aide à la reconversion du salarié - qu'il s'agisse par exemple d'une «reconnaissance de qualité de travailleur handicapé» (RQTH) ou d'une action de maintien dans l'emploi. L'ensemble de ces tâches est effectué au cours du «tiers-temps» (voir encadré 3).

Ces activités administratives, plus fréquentes lors des visites de reprise, de préreprise ou occasionnelles (voir graphique 3), transforment l'activité médicale et le contenu des actions en entreprises. Les médecins sont surchargés d'un travail plus complexe et doivent gérer, tout comme les guichetiers décrits par DuboIs (ibid, p. 109), «deux exigences contradictoires: améliorer leur productivité pour faire face à l'afflux de visiteurs [...] et en même temps accorder plus d'attention (et donc plus de temps) à ces nouveaux visiteurs "à problèmes"».

(36) Décret du 30 janvier $2012 \mathrm{n}^{\circ}$ 2012-135, article R. 462431, op. cit.
Tout comme les tâches administratives supplémentaires qui en découlent, la forme et le contenu de la visite médicale changent également selon sa catégorie. Les visites les plus longues, pour lesquelles les médecins anticipent la rencontre d'un public particulier, sont placées en fin de vacation ou pendant le tiers-temps afin de pouvoir dépasser les 20 minutes habituellement imparties ${ }^{(37)}$. Il s'agit surtout de visites de préreprise ou à la demande des salariés qui y exposent longuement des cas de «souffrance au travail». Elles durent une heure environ.

Mais la durée n'est pas la seule variable d'ajustement. Du type de visite médicale s'ensuit la manière de mener l'entretien et les thèmes qui $\mathrm{y}$ sont abordés. Les visites périodique et d'embauche sont l'occasion d'interroger le salarié sur son état de santé et ses conditions de travail avant de l'examiner physiquement. Lors de la visite d'embauche, après avoir retracé avec le salarié son parcours professionnel, le médecin le questionne sur les risques liés à son métier alors qu'en visite périodique, il se renseigne avant tout sur l'ambiance ou l'organisation du travail au sein de l'entreprise (voir encadré 2). En revanche, en visites de préreprise et de reprise, ce sont les problèmes de santé ayant motivé l'arrêt de travail ou le motif de la prise de rendez-vous de visite occasionnelle qui sont traités en premier avant d'en examiner le lien avec le travail (voir encadré 3).
(37) Les visites périodiques et d'embauche sont les plus courtes et durent généralement de 20 à 30 minutes. 


\section{Encadré 2 \\ Une visite périodique}

La visite se déroule au sein d'une entreprise employant deux cents salariés environ. La médecin du travail y dispose d'un cabinet dans l'ancienne infirmerie. Cette femme dynamique d'une quarantaine d'années suit depuis plusieurs années cette entreprise à laquelle un temps médical spécifique est réservé du fait de sa taille. La médecin la qualifie de «bulle d'oxygène" car c'est le seul endroit où elle fait encore régulièrement des visites périodiques et de la prévention. En visite périodique, la connaissance qu'elle a déjà des postes de travail lui évite de poser certaines questions pour comprendre le travail des salariés.

La deuxième visite de la matinée commence avec un quart d'heure de retard vers $9 \mathrm{~h} 30$. C'est une visite périodique pour un opérateur d'une cinquantaine d'années qui travaille au conditionnement. La médecin va le chercher dans la salle d'attente tout en récupérant les examens complémentaires qu'il vient d'effectuer auprès de son assistante. Cet homme trapu, en vêtements de travail et chaussures de sécurité, parle facilement de son travail. La médecin commence par me présenter puis, après qu'il a accepté ma présence et s'est assis face à son bureau, elle lui demande comment il va et comment se passe son travail. Le salarié se plaint de devoir changer régulièrement de ligne de production, comme toute son équipe. Au moins une semaine par mois, ils vont en renfort à «l'embouteillage» quand leur propre ligne est moins sollicitée. Le travail y est beaucoup plus statique et ennuyeux. La médecin en convient et ajoute que les tâches les plus fatigantes et répétitives sont généralement attribuées aux salariés qui viennent apporter de l'aide. Le salarié déclare avoir davantage mal au dos suite à ce travail. La médecin lui demande alors si ces changements de poste sont prévus longtemps à l'avance, d'un mois sur l'autre ou d'une semaine sur l'autre : les ouvriers le savent souvent deux semaines avant.

Tout en discutant, la médecin écrit ces informations sur une feuille volante qu'elle joint au dossier médical. Elle interroge ensuite le salarié sur son état de santé, en suivant un ordre prédéfini de questions dont elle note les réponses face aux items inscrits sur sa feuille. Après avoir actualisé quelques renseignements d'ordre général, notamment sa situation familiale, la médecin commence par lui demander d'une manière expéditive s'il fait du sport, s'il fume, s'il boit (“L'apéro: c'est le soir, le week-end ?») et s'il prend des médicaments. Elle consigne ensuite au verso les renseignements d'ordre sanitaire après avoir fait préciser au salarié s'il a été arrêté ou s'il a eu des soucis de santé depuis la dernière visite. Comme ce n'est pas le cas, elle le questionne sur ses «épaules, dos, articulations» : le coude droit du salarié est douloureux depuis un mois; puis elle l'interroge sur son «sommeil, appétit, moral».

À l'issue de ces questions, la médecin invite le salarié à se déshabiller dans la petite pièce jouxtant le cabinet et termine son compte rendu. Le salarié revient en sous-vêtements : il porte un bas de contention à la jambe droite. La médecin du travail lui demande s'il le porte constamment ou occasionnellement et le fait s'allonger sur la table d'examen avant de prendre sa tension. Puis elle lui dit : «Alors, faites-moi voir ce coude!» II lui décrit ce qu'il ressent pendant qu'elle le palpe mais elle n'observe aucun signe clinique particulier. Elle lui conseille d'aller faire la radiographie pour laquelle il a déjà un rendez-vous afin de parvenir à un diagnostic qu'un simple examen clinique ne permet pas, et de voir ensuite avec son médecin traitant. La médecin reprend le cours de son examen conventionnel. Elle écoute son cœur au stéthoscope et lui palpe l'abdomen. Puis elle lui fait plier les genoux et lève ses jambes à la verticale avant de lui demander de s'asseoir. Elle place son stéthoscope à l'arrière de sa cage thoracique en lui demandant de respirer fortement, palpe ses trapèzes et le fait déglutir en lui touchant la gorge. Elle le fait s'asseoir sur le bord de la table d'examen avant de vérifier ses réflexes aux genoux. Enfin, elle lui demande de se lever et de toucher le sol avec ses mains pendant qu'elle observe son dos. Le salarié peut ensuite se rhabiller pendant que la médecin remplit le dossier médical. Elle me précise que son internat au CHU (centre hospitalier universitaire) l'a habituée à examiner les salariés de la tête aux pieds.

Lorsque le salarié revient dans le cabinet en finissant de se rhabiller, la médecin le questionne sur l'ambiance au sein de son équipe. Selon lui, «ça va». Elle lui demande si les tensions suite au départ à la retraite de l'ancien chef d'équipe se sont apaisées (1). Le salarié reste évasif. Elle lui dit qu'avec ses collègues, en tant qu'anciens de l'entreprise et détenteurs du savoir-faire, ils doivent faire attention à intégrer les nouveaux. Mais selon lui, ça va mieux à présent, ils ont appris à travailler ensemble, bien qu'il parle de son chef d'équipe et de ses "petits collègues» d'un ton un peu dédaigneux.

La discussion se poursuit, le salarié dit attendre la retraite pendant que la médecin prépare les renseignements administratifs de la fiche d'aptitude (intitulé du poste, type de visite médicale). Comme pour tout salarié qu'elle considère apte, elle s'exclame : «Bon au service!», avec un sourire, avant de remplir la fiche. Le salarié est déçu : "Ah bon? Vous n'allez pas me trouver quelque chose ?» Elle lui propose de mettre une restriction pour qu'il n'ait pas à aller sur les autres lignes de production en cas de sous-activité sur la sienne, mais il ne veut pas être privilégié par rapport à ses collègues. La médecin précise qu'il ne s'agit pas de ça; ils sont là pour parler de santé et ces restrictions ont pour but de préserver la sienne en lui faisant faire des choses moins difficiles. Elle ajoute qu'il est important de partir à la retraite sur ses deux pieds. Elle le déclare finalement "Apte à son poste, port de charge et position statique "fortement déconseillés" " (ils étaient "à éviter" sur la fiche précédente) en ajoutant qu'elle

(1) Elle m'expliquera par la suite que de jeunes chefs d'équipe sont arrivés sur plusieurs lignes de production; s'ils s'entendent bien entre eux, le courant n'est pas forcément passé avec les ouvriers. 
ne met rien d'obligatoire pour laisser de la marge de manœuvre dans la négociation avec le chef de ligne. Après avoir tamponné la fiche pour l'employeur et celle du salarié, elle lui propose de la photocopier à son supérieur hiérarchique direct. II accepte. Pour cette entreprise, elle garde les fiches destinées à l'employeur qu'elle remet directement «là haut » à la fin de la vacation, plutôt que de les remettre aux salariés.

Le salarié suit la médecin dans le bureau de son assistante pour récupérer sa photocopie. La visite n'a pas duré plus de 30 minutes.

(Notes de terrain, observation réalisée le 22 novembre 2011.)

Chaque médecin du travail a sa propre manière de réaliser un examen clinique. Certains se contentent du strict minimum en prenant la tension; d'autres se livrent à un examen complet de l'état des salariés (voir encadré 2). Ces examens sont effectués systématiquement en visite périodique ou d'embauche; mais remplacés par l'étude de la lésion voir supprimés lors des visites de reprise, de préreprise ou occasionnelle (voir encadré 3 ). De manière a priori paradoxale, les médecins du travail examinent de façon moins complète les salariés qui ont des problèmes de santé avérés que ceux qui semblent en bonne santé. Comme ils ne peuvent prescrire de traitements, ils partagent le suivi des pathologies déclarées avec les médecins généralistes et spécialistes. Cette particularité de la spécialité participe d'ailleurs de sa dépréciation dans le champ médical : dans le classement des disciplines médicales par ordre de préférence des étudiants, elle figure bonne dernière ${ }^{(38)}$.

\section{Encadré 3}

\section{Une salariée déclarée inapte}

La salariée "observée " est agent d'entretien pour deux entreprises de nettoyage. Cette femme petite et corpulente a presque 60 ans. II est indiqué sur la feuille de vacation qu'elle a 58 ans mais la médecin du travail, une femme d'une cinquantaine d'années, pense qu'elle triche car elle semble physiologiquement plus âgée.

Notre première rencontre a lieu au cours d'une visite de reprise après maladie. Après s'être installée et après que la médecin du travail lui a demandé ce qui l'amenait, elle explique qu'elle a été arrêtée pour des problèmes d'hypertension (sa tension n'est pas redescendue pendant un mois) et qu'elle a également d'importantes douleurs dans le dos et l'épaule. La médecin (qui suit de nombreuses entreprises de nettoyage et pose systématiquement ces mêmes questions aux agents d'entretien dont le travail se caractérise par une multitude de contrats de courte durée sur des lieux différents, à des horaires atypiques) l'interroge ensuite sur ses conditions de travail, notamment les lieux (sur quels «chantiers» travaille-t-elle ?) et les horaires. Les journées de la salariée sont discontinues : elle commence tôt le matin pour une entreprise et finit tard le soir pour une autre. Son contrat du soir ne lui pose pas de difficultés particulières; en revanche le matin, les horaires et parcours des bus ayant été modifiés, elle doit se lever très tôt et marcher davantage pour prendre sa correspondance. Cette situation est d'autant plus compliquée à vivre pour elle qu'elle dort assez mal et que ses articulations sont moins mobiles le matin. La médecin du travail l'examine ensuite de manière assez détaillée, la questionne sur sa santé (l'amplitude de ses mouvements, la fatigue qu'elle ressent aux différents moments de la journée) et les tâches qu'elle doit effectuer.

À l'issue de l'examen, la médecin retourne derrière son bureau et demande le nom de l'entreprise qui l'emploie le matin afin de rechercher qui en est le médecin du travail au sein du service. Comme cet établissement n'est actuellement suivi par aucun médecin, elle décide de s'occuper de son cas. Elle cherche ensuite à savoir si la salariée a déjà déposé une demande d'invalidité auprès de la Sécurité sociale, ce qui lui semble complètement justifié dans sa situation. Comme la réponse est négative, la médecin rédige un courrier au médecin traitant de la salariée pour qu'il en fasse la requête et explique à cette dernière qu'elle devra voir le médecin-conseil et prouver ses problèmes de santé. Elle la rassure en disant qu'étant donné leur nature, la reconnaissance d'invalidité ne devrait pas poser de problème. La salariée précise que son médecin traitant pensait que c'était au médecin du travail de s'occuper de ces démarches. Tel n'est pas le cas et la docteure déclare que la salariée pourra peut-être s'arranger pour se faire licencier à l'amiable par l'entreprise du matin; si cela n'est pas possible, elle pourra éventuellement la déclarer inapte. Pour l'heure, elle la note "apte" avec des restrictions sur les horaires matinaux (qui sont à éviter avant 10 heures) et sur le port de charges lourdes et m'envoie demander à son assistante, qui est partie déjeuner, une fiche d'aptitude au nom de la deuxième entreprise.

La visite, qui a duré 35 minutes, empiète sur la fin de la vacation. Les collègues de la médecin l'appellent pour savoir si elle les rejoint déjeuner. Une fois sortie du cabinet, elle commente : "On avait bien besoin de ça en fin de matinée!»

(38) Voir FAUVET (2010) et, plus particulièrement, le tableau 3 : «Classement des disciplines dans l'ordre de préférences des étudiants», p. 6. 
La médecin consacre son «tiers-temps» de l'après-midi aux tâches administratives après avoir effectué trois visites médicales (d'embauches et “occasionnelle» à sa propre demande). Elle appelle l'entreprise employant la salariée vue dans la matinée pour expliquer que la fiche d'aptitude délivrée au nom de l'autre employeur les concerne également. Elle propose de la leur faxer pour qu'ils prennent connaissance de son contenu mais ils souhaitent une fiche à leur propre nom. La médecin va donc revoir la salariée en visite afin d'établir une nouvelle fiche dont elle remettra l'original à l'employeur et une copie à la salariée. Elle m'explique ensuite que la situation est d'autant plus compliquée que l'inaptitude envisagée concerne une entreprise qui ne fait pas partie de son «secteur».

Je rencontre de nouveau la salariée trois mois plus tard lors d'une visite de reprise. Entre-temps, elle a été reconnue invalide «deuxième catégorie» par le médecin-conseil de la Sécurité sociale et a accepté l'inaptitude proposée par la médecin du travail. Il s'agit cette fois de la « deuxième visite » qui permet de régler les détails administratifs de l'inaptitude. Une fois que la salariée s'est installée et que nous nous sommes reconnues, la médecin discute directement avec elle de cette décision. Elle ne procède à aucun examen physique.

L'inaptitude va être déclarée pour le «chantier» du matin. C'est l'entreprise où «ça se passait le mieux» mais les horaires de travail restent inadaptés. La négociation de l'inaptitude avec l'employeur a été difficile. La médecin du travail dit avoir reçu une lettre «incendiaire» dans laquelle il remet en question cette décision motivée selon lui par le fait que la salariée ne peut pas prendre le bus; la médecin insiste en expliquant à la salariée qu'elle n'est pas déclarée inapte pour cette raison mais bien à cause de problèmes de santé qui l'empêchent de se déplacer et de travailler. Elle lui répète par ailleurs qu'elle peut la déclarer inapte à son autre poste de travail dès qu'elle le souhaite. Mais la salariée désire le conserver car elle y a «ses copines» et craint avant tout de se retrouver isolée chez elle après avoir été active toute sa vie.

La médecin explique les étapes du licenciement pour lequel la salariée doit rencontrer son employeur au cours de «l'entretien préalable». Elle lui conseille ensuite de faire transférer son dossier de Pôle emploi à un organisme spécialisé dans l'emploi des personnes handicapées (Cap Emploi) afin d'être mieux suivie et lui demande si son entreprise a contracté une prévoyance (1) mais la salariée n'en a aucune idée. Elle l'incite à se renseigner car cela compléterait sa pension d'invalidité.

Elles discutent de l'éventualité d'une nouvelle recherche d'emploi de la salariée et la médecin l'interroge sur ses intentions. Tout en réfléchissant à un emploi qui ne soit pas contre-indiqué par son handicap, elle évoque l'idée d'un travail de surveillance en cantine scolaire. La salariée reprend l'idée en disant que ça lui plairait de s'occuper d'enfants.

En fin de visite, la médecin déclare la salariée «inapte à son poste et à tout poste dans l'entreprise », lui remet son feuillet et garde celui destiné à l'employeur qu'elle lui fera envoyer par recommandé. Puis elle salue la salariée et lui souhaite bon courage. La visite a duré 20 minutes.

(Notes de terrain, observations réalisées les 28 octobre 2011 et 9 février 2012.)

(1) En complément des prestations de la Sécurité sociale, les organismes de prévoyance assurent les salariés en cas de maladie, d'incapacité de travail, d'invalidité, de dépendance et de décès.

Nous voyons que la catégorisation des visites médicales prévue par le Code du travail a des effets concrets sur l'activité des médecins, tant sur son organisation que sur le contenu même des visites. C'est pourquoi les médecins consultent systématiquement, avant chaque vacation, leurs plannings afin d'anticiper la nature des visites programmées.

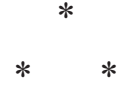

La réforme de la médecine du travail en «santé au travail» ainsi que la division du travail qui découle du recrutement de nouveaux professionnels dans les services interentreprises ont contribué à modifier l'activité et les pratiques médicales. Du fait de la «pénurie médicale» et de décisions législatives, les visites médicales sont redistribuées entre professionnels et les médecins sont amenés à délaisser le suivi régulier de l'ensemble des salariés pour se focaliser sur ceux à la santé plus précaire. Ils rencontrent ces travailleurs, davantage exposés à de mauvaises conditions de travail et d'emploi, principalement lors de visites d'embauche, de reprise après arrêt de travail ou occasionnelle. Cette nouvelle répartition modifie les pratiques médicales, qui varient selon le type de visite effectué. Les médecins se livrent ainsi à un suivi plus complexe fait de visites plus longues et axées sur les problèmes de santé déclarés, assorties d'un suivi administratif et d'actions en entreprise davantage tournés vers la gestion de ces problèmes. Ces pratiques sont notamment celles associées aux déclarations d'inaptitude, dont l'augmentation atteste la prise en charge croissante d'un public à la santé précaire par les médecins du travail. Mais cette nouvelle division du travail qui prévaut dans les services interentreprises n'induit pas une revalorisation du métier de médecin dans la configuration actuelle du champ de la santé au travail. 


\section{Bibliographie}

BACHeT D. (2011), «Des pratiques professionnelles sous tension», Actes de la recherche en sciences sociales, $\mathrm{n}^{\circ} 188$, pp. 54-69.

BASZANGer I. (1986), «Les maladies chroniques et leur ordre négocié», Revue française de sociologie, vol. 1, $\mathrm{n}^{\circ} 27$, pp. 3-27.

Bourdieu P. ([1972] 2000), Esquisse d'une théorie de la pratique, Paris, Seuil.

Bruno A.-S., Geerkens É., Hatzfled N., Omnès C. (dir.) (2011), La santé au travail, entre savoirs et pouvoirs (XIX ${ }_{-}$ XXe siècles), Rennes, Presses universitaires de Rennes.

Buzzi S., Devinck J.-P., Rosental P.-A. (2006), La santé au travail : 1880-2006, Paris, La Découverte.

Castel R. ([1995] 1999), Les métamorphoses de la question sociale: une chronique du salariat, Paris, Gallimard.

Carricaburu D., Ménoret M., ([2004] 2005), Sociologie de la santé. Institutions, professions, maladies, Paris, Armand Colin, 2e édition.

CÉlÉRIER S. (2008), «Santé précaire: quelques perspectives sociologiques », Connaissance de l'emploi, no 56 , Centre d'études de l'emploi.

Caisse nationale de L'assurance-maladie des TRAVAILlEURS SALARIÉS (CNAMTS) (2009), Convention d'objectifs et de gestion pour la branche AT/MP 20092012, Paris.

Dejours C. (1998), Souffrance en France, Paris, Seuil.

Dobry M. (1986), «La régression vers les habitus», in Dobry M., Sociologie des crises politiques. La dynamique des mobilisations multisectorielles, Paris, Presses de la FNSP, pp. 239-259.

Dubois V. ([1999] 2010), La vie au guichet. Relation administrative et traitement de la misère, Paris, Economica, $3 \mathrm{e}$ édition.

FAuvet L. (2010), «Les affectations des étudiants en médecine à l'issue des épreuves classantes nationales en $2009 »$, Études et résultats, $\mathrm{n}^{\circ} 720$, Drees.

Gollac M., Volkoff S. (2007), Les conditions de travail, Paris, La Découverte.

Gollac M. (2012), «Les risques psychosociaux au travail : d'une "question de société" à des questions scientifiques. Introduction», Travail et emploi, $\mathrm{n}^{\circ}$ 129, pp. 5-10.

Hélardot V. (2005), «Précarisation du travail et de l'emploi : quelles résonances dans la construction des expériences sociales ?», Empan, n 60, pp. 30-37.

Hélardot V. (2008), «Enjeux d'emploi, de travail et de santé : la quadrature du cercle», in De Terssac G., SaintMartin C., Thébault C. (dir.), La précarité : une relation entre travail, organisation et santé, Toulouse, Octarès, pp. 73-84.

HuEz D. (1997), «La précarisation de la santé au travail», in Appay B., Thébaud-Mony A. (dir.), Précarisation sociale, travail et santé, Paris, Iresco, pp. 17-26.

Hughes E. (1996), Le regard sociologique: essais choisis, Paris, EHESS.

InAN C. (2013), «Les absences au travail des salariés pour raisons de santé : un rôle important des conditions de travail», Dares analyses, $\mathrm{n}^{\circ} 9$.

Lagarenne C., Lamarche P. (2008), «L'usage des contrats de travail», L'emploi, nouveaux enjeux, Paris, Insee, pp. 51-59.

LIPSKY M. ([1980] 2010), Street-level bureaucracy, $30^{\text {th }}$ anniversary expanded edition, Russel Sage Foundation, New-York.

Loriol M. (2011), "Pourquoi tout ce stress ?», in Lallement M. et al. (dir.), «Maux du travail : dégradation, recomposition ou illusion ?», Sociologie du travail, vol. $53, \mathrm{n}^{\circ} 1$, pp. 9-14.

Lomba C. (2008), «Avant que les papiers ne rentrent dans les cartons : usages ethnographiques des documents d'entreprise", in Arborio A.-M. et al. (dir.), Observer le travail: histoire, ethnographie, approches combinées, Paris, La Découverte, pp. 29-44.

Manuy A., Nouël de la Buzonnière C. (2011), «Une photographie du marché du travail en 2009. Résultats de l'enquête Emploi», Insee première, n 1331.

MARChAND O. (2010), «50 ans de mutations de l'emploi», Insee Première, $\mathrm{n}^{\circ} 1312$.

Marichalar P. (2010), «La médecine du travail sans les médecins ? Une action patronale de longue haleine (1971-2010)», Politix, vol. 3, n 91, pp. 27-52.

Marichalar P. (2011), Prévenir ou produire. Autonomie et subordination dans la médecine du travail (France 1970-2010), Thèse de doctorat en sociologie, Paris, EHESS

OMnÈs C., BRuno A.-S. (dir.) (2004), Les mains inutiles. Inaptitude au travail et emploi en Europe, Paris, Belin.

Peneff J. (1992), L’hôpital en urgences, Paris, Métailié.

SPIRE A. (2008), Accueillir ou reconduire. Enquête sur les guichets de l'immigration, Paris, Raisons d'agir.

STRAuss A. (1992), La trame de la négociation : sociologie qualitative et interactionnisme, Paris, L'Harmattan.

ThéBAud-Mony A. (1997), «Le rapport santé-travail : instrument et enjeu de la précarisation sociale», in Appay B., Thébaud-Mony A. (dir.), Précarisation sociale, travail et santé, Paris, Iresco, pp. 555-575. 